\title{
WEAK TOPOLOGIES FOR THE CLOSED SUBSETS OF A METRIZABLE SPACE
}

\author{
GERALD BEER AND ROBERTO LUCCHETTI
}

\begin{abstract}
The purpose of this article is to propose a unified theory for topologies on the closed subsets of a metrizable space. It can be shown that all of the standard hyperspace topologies-including the Hausdorff metric topology, the Vietoris topology, the Attouch-Wets topology, the Fell topology, the locally finite topology, and the topology of Mosco convergence-arise as weak topologies generated by families of geometric functionals defined on closed sets. A key ingredient is the simple yet beautiful interplay between topologies determined by families of gap functionals and those determined by families of Hausdorff excess functionals.
\end{abstract}

\section{INTRODUCTION}

From the point of view of analysis, the favorite topology for the (nonempty) closed and bounded subsets of a metric space $\langle X, d\rangle$-especially the closed and bounded convex subsets of a normed linear space-is the Hausdorff metric topology. For $A$ and $B$ closed and bounded, the Hausdorff distance between them is defined by

$$
\begin{aligned}
H_{d}(A, B) & =\max \left\{\sup _{a \in A} d(a, B), \sup _{b \in B} d(b, A)\right\}, \\
& =\inf \left\{\varepsilon>0: A \subset S_{\varepsilon}[B] \text { and } B \subset S_{\varepsilon}[A]\right\},
\end{aligned}
$$

where $S_{\varepsilon}[F]$ is the $\varepsilon$-enlargement of the set $F$, i.e., $S_{\varepsilon}[F]=\{x \in X: d(x, F)$ $<\varepsilon\}$. Denoting the Hausdorff excess [CV] $\sup _{b \in B} d(b, A)$ of $B$ over $A$ by $e_{d}(B, A)$, we may write $H_{d}(A, B)=\max \left\{e_{d}(A, B), e_{d}(B, A)\right\}$.

The Hausdorff distance so defined makes sense for arbitrary closed sets as well, and yields an infinite valued metric on the nonempty closed subsets $C L(X)$ of $X[\mathrm{CV}, \mathrm{KT}]$. For closed sets we have the formula [Co]

$$
H_{d}(A, B)=\sup _{x \in X}|d(x, A)-d(x, B)|,
$$

so that Hausdorff metric convergence of a sequence of closed sets $\left\langle A_{n}\right\rangle$ to $A$ amounts to the uniform convergence of $\left\langle d\left(\cdot, A_{n}\right)\right\rangle$ to $d(\cdot, A)$.

Received by the editors November 16, 1990.

1980 Mathematics Subject Classification (1985 Revision). Primary 54B20; Secondary $28 \mathrm{~A} 10$.

Key words and phrases. Hyperspace, gap functional, Hausdorff excess, Hausdorff metric topology, Attouch-Wets topology, Wijsman topology, Vietoris topology, distance functional, weak topologies.

Research of the first author was partially supported by NSF Grant DMS- 9001096.

Research of the second author was partially supported by MPI grant, Italy. 
But there is a problem with this metric in the more general setting: it is obviously too strong. In the plane, one would like the sequence of lines $\left\langle L_{n}\right\rangle$ where $L_{n}$ has slope $1 / n$ and $y$-intercept 0 to converge to the $x$-axis. This, of course, fails for the Hausdorff metric induced by the Euclidean metric. Various weaker convergence notions/topologies for closed sets have been considered over the past thirty years, with variable success: Kuratowski convergence and the associated Fell topology; Wijsman convergence (pointwise convergence of $\left\langle d\left(\cdot, A_{n}\right)\right\rangle$ to $d(\cdot, A)$ ) and the associated Wijsman topology; Mosco convergence and the associated Mosco topology. Only recently has a completely acceptable replacement (at least in the convex case) for the Hausdorff metric been investigated: the metrizable topology of uniform convergence of $\left\langle d\left(\cdot, A_{n}\right)\right\rangle$ to $d(\cdot, A)$ on bounded subsets of $X$. Given $x_{0} \in X$, a local base for this topology [Be2, BDC, AP, ALW] at $A \in C L(X)$ consists of all sets of the form

$$
\begin{array}{r}
\Sigma_{n}[A] \equiv\left\{F \in C L(X): F \cap S_{n}\left[x_{0}\right] \subset S_{1 / n}[A] \text { and } A \cap S_{n}\left[x_{0}\right] \subset S_{1 / n}[F]\right\} \\
\left(n \in Z^{+}\right) .
\end{array}
$$

In the setting of convex analysis, this topology reduces to the Hausdorff metric topology for closed and bounded convex sets [BL1], is stable with respect to duality [ $\mathrm{Be} 3, \mathrm{Pe}]$, and is well suited for approximation and optimization. In view of its seminal study in [AW], we call this the Attouch-Wets topology $\tau_{\mathrm{aw}_{d}}$, although it has been often called the bounded Hausdorff topology [AP, Pe].

In the last few years, a significant development in the study of topologies on the closed subsets of a metric space has been the presentation of many basic topologies as weak topologies. Given a topology $\tau$ on $C L(X)$, one seeks a family $\left\{\psi_{i}: i \in I\right\}$ of extended real functionals on $C L(X)$ such that $\tau$ is the weakest topology for which each $\psi_{i}$ is continuous. Here are some typical results within this general framework.

If $X$ is a metrizable space and $\left\{d_{i}: i \in I\right\}$ is the family of all compatible metrics, then the Vietoris topology [Mi, KT, En] is the weakest topology $\tau$ on $C L(X)$ such that for each $x \in X$ and $i \in I, A \rightarrow d_{i}(x, A)$ is $\tau$-continuous [BLLN, Theorem 3.1]. Thus the Vietoris topology is the weak topology determined by $\left\{d_{i}(x, \cdot): x \in X\right.$ and $\left.i \in I\right\}$. The Fell topology [At, Fe, KT] on $C L(X)$ is Hausdorff if and only if $X$ is locally compact, and in this context there exists a compatible metric $d$ (specifically, one such that each closed $d$ ball that is a proper subset of $X$ is compact) such that the Fell topology is the Wijsman topology determined by $d$, i.e., the weak topology generated by $\{d(x, \cdot): x \in X\}$ [Be4, Theorem 2]. The topology of Mosco convergence on the closed convex subsets of a Banach space $X$, compatible with Mosco convergence [At, Mo, BF] of sequences [Be1, Theorem 3.1] is Hausdorff if and only if $X$ is reflexive [BB]. In this case, the Mosco topology is the weak topology generated by the family of functionals $\left\{D_{d}(\cdot, K): K\right.$ weakly compact and convex [Bel, Theorem 3.3], where $d$ is the distance functional associated with the norm of $X$, and

$$
D_{d}(A, B)=\inf \{d(a, b): a \in A, b \in B\}=\inf _{b \in B} d(b, A),
$$

is the gap between two closed sets $A$ and $B$ relative to the metric $d$. Moreover, there exists a renorming of $X$ with associated metric $d$ (one such that the dual norm has the Kadec property) such that the Mosco topology is generated by 
$\{d(x, \cdot): x \in X\}$ [BF, Be7]. If $X$ is an arbitrary normed linear space, then the weak topology on the closed convex sets determined by $\left\{D_{d}(\cdot, C): C\right.$ closed and convex $\}$ coincides with the weak topology determined by $\{d(x, \cdot): x \in$ $X\} \cup\left\{s(y, \cdot): y \in X^{*}\right\}$, where $s(y, A)=\sup \{\langle y, a\rangle: a \in A\}$ is the value of the support functional for the set $A$ at $y$ [Be5]. The weak topology determined by support functionals alone on the closed convex sets alone is studied in [SZ1].

Here, we systematically study topologies on $C L(X)$ induced by gap functionals and excess functionals where one set argument is fixed, varying both the set and metric over prescribed classes. In particular, we show that the Hausdorff metric and Attouch-Wets topologies both fit within this framework in a similar way. One point of departure for this work is the $d$-proximal topology $\tau_{\delta_{d}}$ on $C L(X)$ introduced in [BLLN], which may be defined as the weak topology on $C L(X)$ determined by the family of gap functionals $\left\{D_{d}(\cdot, F): F \in C L(X)\right\}$. A second point of departure is a certain weakening $\sigma_{d}$ of $\tau_{\mathrm{aw}_{d}}$, considered recently in the context of convex analysis and optimization, by two different sets of authors [AAB, SP].

Definition. The bounded d-proximal topology $\sigma_{d}$ on $C L(X)$ has as a local base at $A \in C L(X)$ all sets of the form

$$
\begin{aligned}
& \Phi_{A}\left[n ; a_{1}, a_{2}, \ldots, a_{k}\right] \equiv\left\{F \in C L(X): F \cap S_{n}\left[x_{0}\right] \subset S_{1 / n}[A],\right. \\
&\text { and } \left.\forall i \leq k, d\left(a_{i}, F\right)<1 / n\right\}
\end{aligned}
$$

where $\left\{a_{1}, a_{2}, \ldots, a_{k}\right\}$ is a finite subset of $A$ and $n \in Z^{+}$.

As we shall see, this topology is the weak topology determined by $\left\{D_{d}(\cdot, B)\right.$ : $B \in C L(X)$ and $B$ bounded\}, a result which has been obtained independently and concurrently in sequential form by Sonntag and Zalinescu [ZS2] (private communication). We then form natural duals for the $d$-proximal and bounded $d$-proximal topologies, both in terms of their local presentations and in terms of the lattice-theoretic approach to hyperspaces as promoted by Levi and Lechicki [FLL, LL], and show that these topologies are weak topologies determined by Hausdorff excess functionals. Putting these together yields the Hausdorff metric and Attouch-Wets topologies.

\section{Preliminaries}

As stated in $\S 1, C L(X)$ will denote the nonempty closed subsets of a metric space $\langle X, d\rangle$. We need to review some basic facts about hyperspace topologies, i.e., topologies on $C L(X)$. In view of the results mentioned in $\S 1$, a basic topology on $C L(X)$ is the Wijsman topology $\tau_{W_{d}}$ [Wi, Co, FLL, LL, BLLN, Be6], which is the weakest topology $\tau$ on $C L(X)$ such that for each $x \in X$, the functional $A \rightarrow d(x, A)$ is $\tau$-continuous. This topology is a function space topology, in that $\tau_{W_{d}}$ is the topology that $C L(X)$ inherits from $C(X, R)$, equipped with the topology of pointwise convergence, under the identification $A \leftrightarrow d(\cdot, A)$. Similarly, the Hausdorff metric (resp. Attouch-West) topology is the topology that $C L(X)$ inherits from $C(X, R)$, equipped with the topology of uniform convergence (resp. uniform convergence on bounded sets), under the identification $A \leftrightarrow d(\cdot, A)$.

Another basic class of hyperspace topologies are the hit-and-miss topologies. To introduce these, we need some notation. For $E \subset C L(X)$, we introduce the 
following subsets of $C L(X)$ :

$$
\begin{aligned}
E^{-} & \equiv\{A \in C L(X): A \cap E \neq \varnothing\} \\
E^{+} & \equiv\{A \in C L(X): A \subset E\} \\
E^{++} & \equiv\left\{A \in C L(X): \text { there exists } \varepsilon>0 \text { with } S_{\varepsilon}[A] \subset E\right\} .
\end{aligned}
$$

A set in $E^{-}$hits $E$, whereas a set in $E^{+}$misses $E^{c}$. A set in $E^{++}$really misses $E^{c}$ ! Using this notation, we list some standard hit-and-miss topologies:

(1) The Vietoris topology $\tau_{V}[\mathrm{Mi}, \mathrm{KT}, \mathrm{En}]$ on $C L(X)$ has as a subbase all sets of the form $V^{-}$where $V$ is open, and all sets of the form $W^{+}$ where $W$ is open;

(2) The Fell topology $\tau_{F}[\mathrm{Fe}, \mathrm{KT}]$ on $C L(X)$ has as a subbase all sets of the form $V^{-}$where $V$ is open, and all sets of the form $W^{+}$where $W$ has compact complement;

(3) For $X$ a normed linear space, the Mosco topology $\tau_{M}$ [Be1, BB, Be7] on the weakly closed sets has as a subbase all sets of the form $V^{-}$where $V$ is norm open, and all sets of the form $W^{+}$where $W$ has weakly compact complement.

Notice that in (2) and (3), $W^{+}=W^{++}$for the given class of sets $W$. If in (1), we replace $W^{+}$by $W^{++}$, as $W$ runs over the open sets, we obtain the so-called $d$-proximal topology $\tau_{\delta_{d}}$ studied in [BLLN]. This topology has a presentation as a weak topology, alluded to in $\S 1$ : the $d$-proximal topology is the weakest topology $\tau$ on $C L(X)$ such that for each $F \in C L(X), A \rightarrow D_{d}(A, F)$ is $\tau$-continuous [BLLN, Theorem 3.2]. Analogously, the Mosco topology is the weakest topology $\tau$ on the weakly closed subsets of a reflexive Banach space such that for each weakly compact set $K, A \rightarrow D_{d}(A, K)$ is $\tau$-continuous, where $d$ is the metric induced by the norm [Be1, Theorem 3.3]. These two results are special cases of a general phenomenon that we now describe.

Definition. Let $\Omega$ be a class of nonempty closed subsets of a metric space $\langle X, d\rangle$. We say that $\Omega$ is stable under enlargements if for each $A \in \Omega$ and $\alpha>0$, we have $\operatorname{cl} S_{\alpha}[A] \in \Omega$.

Within $C L(X)$, we distinguish these classes:

$$
K(X)=\text { the nonempty compact subsets; }
$$

$C L B(X)=$ the nonempty closed and bounded subsets;

Evidently, $C L B(X)$ and $C L(X)$ are stable under enlargements, as is $K(X)$ provided closed and bounded subsets of $X$ are compact. In a normed linear space, the convex sets, the connected sets, and the starshaped sets are stable under enlargements. If the space is reflexive, then the weakly compact sets also have this property.

We need to consider semicontinuity of functionals defined on hyperspaces. Let $T$ be a topological space. Recall that $f: T \rightarrow[-\infty, \infty]$ is called lower semicontinuous provided for each $\alpha \in R,\{t: f(t) \leq \alpha\}$, is a closed subset of $T$. We call $f$ upper semicontinuous provided $-f$ is lower semicontinuous.

Theorem 2.1. Let $\langle X, d\rangle$ be a metric space, and let $\Omega$ be a class of closed subsets that is stable under enlargements and that contains the singleton subsets of $X$. Let $\Pi$ be a subset of $C L(X)$. Then the topology $\tau_{1}$ on $\Pi$ having as 
a subbase all sets of the form $V^{-}$where $V$ is open, and all sets of the form $\left(E^{c}\right)^{++}$where $E \in \Omega$, is the weakest topology $\tau$ on $\Pi$ such that for every $E \in \Omega, A \rightarrow D_{d}(A, E)$ is $\tau$-continuous.

Proof. Let $\tau_{\text {weak }}$ be the weak topology so described. We first show $\tau_{\text {weak }} \supset \tau_{1}$. Suppose $A \in \Pi$. If $A \in V^{-}$where $V$ is open, we can find $a \in A$ and $\varepsilon>0$ with $S_{\varepsilon}[a] \subset V$. Then $\{F \in \Pi: d(a, F)<\varepsilon\}=\left\{F \in \Pi: D_{d}(F,\{a\})<\varepsilon\right\}$ is a $\tau_{\text {weak-neighborhood of }} A$, and $\{F \in \Pi: d(a, F)<\varepsilon\}=S_{\varepsilon}[A]^{-} \subset V^{-}$. On the other hand, for each $E \in \Omega$, we have $\left(E^{c}\right)^{++}=\left\{F \in \Pi: D_{d}(F, E)>0\right\} \in$ $\tau_{\text {weak }}$. This proves $\tau_{\text {weak }} \supset \tau_{1}$.

For the other inclusion, it suffices to show that $F \rightarrow D_{d}(F, E)$ is $\tau_{1^{-}}$ continuous on $\Pi$ for each $E \in \Omega$. For upper semicontinuity, fix $A \in \Pi$ and $\varepsilon>0$. Pick $a \in A$ with $d(a, E)<D_{d}(A, E)+\varepsilon / 2$. Then if $F \in\left(S_{\varepsilon / 2}[a]\right)^{-} \cap$ $\Pi$, we have $D_{d}(F, E)<D_{d}(A, E)+\varepsilon$. Lower semicontinuity of the gap functional holds if $D_{d}(A, E)=0$. Otherwise, write $D_{d}(A, E)=\alpha>0$ and let $\varepsilon \in(0, \alpha)$ be arbitrary. Then since $\Omega$ is stable under enlargements, $\left(\left(\operatorname{cl} S_{\alpha-\varepsilon}[E]\right)^{c}\right)^{++}$is a $\tau_{1}$-neighborhood of $A$, and if $F \in\left(\left(\operatorname{cl} S_{\alpha-\varepsilon}[E]\right)^{c}\right)^{++}$, we obtain $D_{d}(F, E) \geq \alpha-\varepsilon$.

For emphasis, we state as corollaries these special cases.

Corollary 2.2. Let $\langle X, d\rangle$ be a metric space. Then the $d$-proximal topology on $C L(X)$, having as a subbase all sets of the form $V^{-}$where $V$ is open, and all sets of the form $W^{++}$where $W$ is open, is the weakest topology $\tau$ on $C L(X)$ such that $A \rightarrow D_{d}(A, F)$ is $\tau$-continuous for each $F \in C L(X)$.

Corollary 2.3. Let $\langle X, d\rangle$ be a reflexive Banach space. Then the Mosco topology $\tau_{M}$ on the nonempty weakly closed subsets of $X$, having as a subbase all sets of the form $V^{-}$where $V$ is norm open, and all sets of the form $W^{+}$where $W$ has weakly compact complement, is the weakest topology $\tau$ on the weakly closed subsets such that $A \rightarrow D_{d}(A, K)$ is $\tau$-continuous for each weakly compact subset $K$ of $X$.

Proof. By weak lower semicontinuity of the norm, for each $\alpha>0$, we have $\operatorname{cl} S_{\alpha}[K]=\{x \in X: d(x, K) \leq \alpha\}$ weakly closed whenever $K$ is weakly compact. Thus by reflexivity, weak compactness of $K$ yields weak compactness of $\operatorname{cl} S_{\alpha}[K]$. Finally, if $A$ is weakly closed, and $K$ is weakly compact and $A \cap K \neq \varnothing$, then $A \in\left(K^{c}\right)^{++}$, again by weak lower semicontinuity of the norm.

\section{LOCAL AND GLOBAL PRESENTATIONS OF $\sigma_{d}$}

Lemma 3.1. Let $\langle X, d\rangle$ be a metrizable space, and let $A \in C L(X)$. The following families also constitute local bases for the bounded d-proximal topology $\sigma_{d}$ at $A$ :

(i) All sets of the form $\boldsymbol{\Theta}_{A}\left[B ; \varepsilon ; a_{1}, a_{2}, \ldots, a_{k}\right]=\{F \in C L(X): F \cap B \subset$ $S_{\varepsilon}[A]$, and $\left.\forall i \leq k, d\left(a_{i}, F\right)<\varepsilon\right\}$, where $\left\{a_{1}, a_{2}, \ldots, a_{k}\right\} \subset A$ and $B$ is bounded;

(ii) All sets of the form $\Lambda_{A}\left[B ; \varepsilon ; x_{1}, x_{2}, \ldots, x_{k}\right]=\{F \in C L(X): \forall x \in$ B,$d(x, A)-\varepsilon<d(x, F)$ and $\left.\forall i \leq k, d\left(x_{i}, F\right)<d\left(x_{i}, A\right)+\varepsilon\right\}$, where $\left\{x_{1}, x_{2}, \ldots, x_{k}\right\} \subset X$ and $B$ is bounded. 
Proof. Let $\left\{a_{1}, a_{2}, \ldots, a_{k}\right\} \subset A$ and let $n \in Z^{+}$be given. With $B=S_{n}\left[x_{0}\right]$ and $\varepsilon=1 / n$, we have $\boldsymbol{\Theta}_{A}\left[B ; \varepsilon ; a_{1}, a_{2}, \ldots, a_{k}\right]=\Phi_{A}\left[n ; a_{1}, a_{2}, \ldots, a_{k}\right]$. Now let $B$ be bounded, $\varepsilon>0$, and $\left\{a_{1}, a_{2}, \ldots, a_{k}\right\} \subset A$ be given. If $F \in \Lambda_{A}\left[B ; \varepsilon ; a_{1}, a_{2}, \ldots, a_{k}\right]$, then for every $x \in F \cap B$, we have $d(x, A)-\varepsilon<$ $d(x, F)=0$ so that $x \in S_{\varepsilon}[A]$. This proves that $\Lambda_{A}\left[B ; \varepsilon ; a_{1}, a_{2}, \ldots, a_{k}\right] \subset$ $\boldsymbol{\Theta}_{A}\left[B ; \varepsilon ; a_{1}, a_{2}, \ldots, a_{k}\right]$. Finally, let $B$ be bounded, $\varepsilon>0$, and points $\left\{x_{1}, x_{2}, \ldots, x_{k}\right\} \subset X$ be given. Choose for each $i \leq k$ a point $a_{i} \in A$ with $d\left(x_{i}, a_{i}\right)<d\left(x_{i}, A\right)+\varepsilon / 2$. There exists $m \in Z^{+}$such that $1 / m<\varepsilon$ and $B \subset S_{m}\left[x_{0}\right]$. Choose $m_{0}>m$ so large that $F \cap S_{m_{0}}\left[x_{0}\right] \neq \varnothing$ for each $F \in \Phi_{A}\left[m_{0} ; a_{1}, a_{2}, \ldots, a_{k}\right]$ (we may for example, take $m_{0}>m$ so large that $\left.S_{m_{0}}\left[x_{0}\right] \supset S_{1}\left[a_{1}\right]\right)$. Set $n=2 m+m_{0}+1$. We claim that $\Phi_{A}\left[n ; a_{1}, a_{2}, \ldots, a_{k}\right]$ $\subset \Lambda_{A}\left[B ; \varepsilon ; x_{1}, x_{2}, \ldots, x_{k}\right]$.

Fix $F \in \Phi_{A}\left[n ; a_{1}, a_{2}, \ldots, a_{k}\right]$. For each $i \leq k$, we have $d\left(a_{i}, F\right)<1 / n<$ $1 / 2 m<\varepsilon / 2$ so that

$$
d\left(x_{i}, F\right) \leq d\left(x_{i}, a_{i}\right)+d\left(a_{i}, F\right)<d\left(x_{i}, A\right)+\varepsilon .
$$

For each $x \in S_{m}\left[x_{0}\right]$ take $z_{x} \in F$ with $d\left(x, z_{x}\right)<d(x, F)+1 / 3 m$. Since $F$ hits $S_{m_{0}}\left[x_{0}\right]$, we have

$$
d\left(x_{0}, z_{x}\right) \leq d\left(x_{0}, x\right)+d\left(x, z_{x}\right)<m+\left(m+m_{0}+1\right)=n .
$$

Since $F \cap S_{n}\left[x_{0}\right] \subset S_{1 / n}[A]$, there exists $a_{x} \in A$ with $d\left(a_{x}, z_{x}\right)<1 / n<1 / 3 m$. Thus, we have

$$
\begin{aligned}
d(x, A) & \leq d\left(x, a_{x}\right) \leq d\left(x, z_{x}\right)+d\left(z_{x}, a_{x}\right) \\
& <d(x, F)+\frac{1}{3 m}+\frac{1}{3 m}<d(x, F)+\frac{2}{3 m} .
\end{aligned}
$$

This proves that $\forall x \in S_{m}\left[x_{0}\right]$, we have $d(x, A)-1 / m<d(x, F)$, whence $\forall x \in$ $B, d(x, A)-\varepsilon<d(x, F)$. This proves that $F \in \Lambda_{A}\left[B ; \varepsilon ; x_{1}, x_{2}, \ldots, x_{k}\right]$.

We intentionally work with the different presentations of $\sigma_{d}$ throughout this paper, as the situation dictates. The next two results are representative scenarios in this regard.

Corollary 3.2. Let $\langle X, d\rangle$ be a metric space. Then $\sigma_{d} \supset \tau_{W_{d}}$.

Proof. Fix $A \in C L(X)$. Given $x \in X$ and $\varepsilon>0, \Lambda_{A}[\{x\} ; \varepsilon ; x]=\{F \in$ $C L(X): d(x, A)-\varepsilon<d(x, F)<d(x, A)+\varepsilon\}=\{F \in C L(X): \mid d(x, F)-$ $d(x, A) \mid<\varepsilon\}$.

As $\tau_{W_{d}}$ is Hausdorff, the same must be true for the stronger $\sigma_{d}$.

Corollary 3.3. Let $\langle X, d\rangle$ be a metric space. Then

(i) $\sigma_{d} \subset \tau_{\mathrm{aw}_{d}}$ on $C L(X)$;

(ii) If $\left\langle A_{\lambda}\right\rangle$ is a net in $C L(X) \sigma_{d}$-convergent to a totally bounded closed set $A$, then $A=\tau_{\text {aw }_{d}}-\lim A_{\lambda}$.

Proof. (i) Fix $x_{0} \in X$, and fix $A \in C L(X)$, and let $\Phi_{A}\left[n ; a_{1}, a_{2}, \ldots, a_{k}\right]$ be a $\sigma_{d}$-neighborhood of $A$. Choose $m>n$ so large that $\left\{a_{1}, a_{2}, \ldots, a_{k}\right\} \subset$ $S_{m}\left[x_{0}\right]$. We clearly have $\Sigma_{m}[A] \subset \Phi_{A}\left[n ; a_{1}, a_{2}, \ldots, a_{k}\right]$.

(ii) Fix $x_{0} \in X$ and $n \in Z^{+}$. By total boundedness, choose $\left\{a_{1}, a_{2}, a_{3}, \ldots\right.$, $\left.a_{k}\right\}$ in $A$ with $A \subset S_{1 / 2 n}\left[\left\{a_{1}, a_{2}, a_{3}, \ldots, a_{k}\right\}\right]$. We then have $\Sigma_{n}[A] \supset$ $\Phi_{A}\left[2 n ; a_{1}, a_{2}, \ldots, a_{k}\right]$. Thus, $\left\langle A_{\lambda}\right\rangle$ must be in $\Sigma_{n}[A]$ eventually, so that $A=\tau_{\mathrm{aw}_{d}}-\lim A_{\lambda}$. 
It can be shown that pairwise coincidence of the topologies $\tau_{\mathrm{aw}_{d}}, \sigma_{d}$, and $\tau_{W_{d}}$ occurs if and only if bounded subsets of $X$ are totally bounded. This fact, as well as necessary and sufficient conditions for first countability, second countability, and metrizability, as well as a description of the properties of the underlying metric that determine the bounded $d$-proximal topology, are presented in [BL2]. As we will introduce several new topologies in this paper, it would be distracting (and tedious) to pursue a complete analysis here.

We now turn to global presentations of $\sigma_{d}$.

Theorem 3.4. Let $\langle X, d\rangle$ be a metric space. The topology $\sigma_{d}$ is the weakest topology $\tau$ on $C L(X)$ such that for each closed and bounded subset $B$ of $X$, the gap functional $A \rightarrow D_{d}(A, B)$ is $\tau$-continuous on $C L(X)$.

Proof. For $B \in C L B(X)$ fixed, we first show that $F \rightarrow D_{d}(F, B)$ is $\sigma_{d^{-}}$ continuous. Fix $A \in C L(X)$ and let $\varepsilon>0$. There exists $a \in A$ such that $d(a, B)<D_{d}(A, B)+\varepsilon / 2$. Now $\Theta_{A}[B ; \varepsilon / 2 ; a]$ is a $\sigma_{d}$-neighborhood of $A$, and $D_{d}(F, B)<D_{d}(A, B)+\varepsilon$ for each $F$ in the neighborhood. This proves $\sigma_{d}$-upper semicontinuity of $F \rightarrow D_{d}(F, B)$ at $A$. Lower semicontinuity is obvious if $D_{d}(A, B)=0$; so, suppose $D_{d}(A, B)=\alpha>0$. Let $\varepsilon \in(0, \alpha)$ and $a_{1} \in A$ be arbitrary. We claim that for each $F$ in the $\sigma_{d}$-neighborhood $\Lambda_{A}\left[S_{\alpha-\varepsilon}[B] ; \varepsilon ; a_{1}\right]$ of $A$, we have $D_{d}(F, B) \geq \alpha-\varepsilon$.

Fix $F \in \Theta_{A}\left[S_{\alpha-\varepsilon}[B] ; \varepsilon ; a_{1}\right]$. We claim that $F \cap S_{\alpha-\varepsilon}[B]$ is empty. Otherwise, taking $x \in F \cap S_{\alpha-\varepsilon}[B]$, we have $x \in S_{\varepsilon}[A]$, so that $A \cap S_{\varepsilon}\left[S_{\alpha-\varepsilon}[B]\right] \neq \varnothing$, contradicting $D_{d}(A, B)=\alpha$. This means that $D_{d}(F, B) \geq \alpha-\varepsilon$, establishing $\sigma_{d}$-lower semicontinuity of the gap functional at $A$. Thus if $\tau_{\text {weak }}$ is the weakest topology $\tau$ on $C L(X)$ such that $F \rightarrow D_{d}(F, B)$ is $\tau$-continuous for each closed and bounded set $B$, we have $\sigma_{d} \supset \tau_{\text {weak }}$.

To show that $\sigma_{d} \subset \tau_{\text {weak }}$, we show that if $\left\langle A_{\mu}\right\rangle$ is a net in $C L(X) \tau_{\text {weak }}{ }^{-}$ convergent to $A$, then $A=\sigma_{d}-\lim A_{\mu}$. To this end, let $\Theta_{A}\left[B ; \varepsilon ; a_{1}, a_{2}, \ldots, a_{k}\right]$ be a $\sigma_{d}$-neighborhood of $A$, where $B$ is bounded and $\left\{a_{1}, a_{2}, \ldots, a_{n}\right\} \subset A$. For each $i \leq k$ we have

$$
0=D_{d}\left(A,\left\{a_{i}\right\}\right)=\lim _{\mu} D_{d}\left(A_{\mu},\left\{a_{i}\right\}\right)=\lim _{\mu} d\left(a_{i}, A_{\mu}\right) .
$$

Thus, there exists an index $\mu_{0}$ such that for each $\mu \geq \mu_{0}$ and each $i \leq k$, we have $d\left(a_{i}, A_{\mu}\right)<\varepsilon$. It remains to show that eventually, $A_{\mu} \cap B \subset S_{\varepsilon}[A]$. Suppose to the contrary that $A_{\mu} \cap B \not \subset S_{\varepsilon}[A]$ for each $\mu$ in some cofinal set of indices $M$. For each $\mu \in M$, pick $x_{\mu} \in A_{\mu} \cap B$ with $d\left(x_{\mu}, A\right) \geq \varepsilon$. Then $B^{\prime}=\operatorname{cl}\left\{x_{\mu}: \mu \in M\right\}$ is closed and bounded and $D_{d}\left(A, B^{\prime}\right) \geq \varepsilon$. However, $\lim _{\mu} D_{d}\left(A_{\mu}, B^{\prime}\right) \geq \varepsilon$ is impossible, since $D_{d}\left(A_{\mu}, B^{\prime}\right)=0$ frequently. This contradicts $A=\tau_{\text {weak }}-\lim A_{\mu}$. Thus, eventually, $A_{\mu} \cap B \subset S_{\varepsilon}[A]$ must hold, and we have shown that eventually $A_{\mu} \in \Theta_{A}\left[B ; \varepsilon ; a_{1}, a_{2}, \ldots, a_{k}\right]$ must hold.

One consequence of Theorem 3.4 is that the topology $\sigma_{d}$ is completely regular, for any weak topology induced by a family of functions into uniform spaces has a natural compatible uniformity. We also remark that in the last theorem, there is really no need to require that the sets $B$ be closed as well as bounded, since for any $B$, we have $D_{d}(A, B)=D_{d}(A, \mathrm{cl} B)$.

Gap functionals determined by a fixed closed argument need not be $\sigma_{d^{-}}$ continuous or even $\tau_{\mathrm{aw}_{d}}$-continuous, as the following example shows. 
Example. In the plane with the usual metric, let $F=\{(x, y): y=1\}$, let $A_{n}=\{(x, y): y=x / n\}$ and let $A=\{(x, y): y=0\}$. Then $A=\tau_{\text {aw }_{d}}-\lim A_{n}$ and $D_{d}(F, A)=1$, whereas for each $n, D_{d}\left(F, A_{n}\right)=0$.

Corollary 3.5. Let $X$ be a reflexive Banach space. Then the bounded d-proximal topology when restricted to the weakly closed nonempty subsets of $X$ is finer than the Mosco topology.

Proof. The Mosco topology is generated by a smaller family of gap functionals, namely those determined by the weakly closed and norm bounded subsets of $X$.

It can be shown that the bounded $d$-proximal topology coincides with the Mosco topology if and only if the underlying space is finite dimensional [BL2].

As a result of Theorems 2.1 and 3.4, we may represent $\sigma_{d}$ as a hit-and-miss topology.

Theorem 3.6. Let $\langle X, d\rangle$ be a metric space. Then a subbase for the bounded $d$-proximal topology consists of all sets of the form $V^{-}$where $V$ is open, and all sets of the form $\left(B^{c}\right)^{++}$, where $B$ is closed and bounded.

In view of Theorems 3.4 and 3.6, the topology $\sigma_{d}$ is indeed an analogue of the $d$-proximal topology $\tau_{\delta_{d}}$ introduced in [BLLN]. Now $\tau_{\delta_{d}}$ is the weakest topology on $C L(X)$ such that $A \rightarrow \rho(x, A)$ is continuous, where $\rho$ ranges over the metrics that define the same uniformity as $d$ and $x$ ranges over $X$ [BLLN, Theorem 3.7]. Put somewhat differently, $\tau_{\delta_{d}}=\sup \left\{\tau_{W_{\rho}}: \rho\right.$ is uniformly equivalent to $d\}$, where the supremum is taken in the lattice of hyperspace topologies. Does $\sigma_{d}$ admit such a presentation? Our next result resolves this affirmatively.

Theorem 3.7. Let $\langle X, d\rangle$ be a metric space and let $\Delta=\{\rho: \rho$ is a metric uniformly equivalent to $d$ that determine the same bounded sets as $d\}$. Then $\sigma_{d}$ is the weak topology on $C L(X)$ determined by $\{\rho(x, \cdot): x \in X, \rho \in \Delta\}$.

Proof. Let $\tau_{\text {weak }}$ be the specified weak topology. Clearly, if $d$ and $\rho$ are uniformly equivalent, then for each $A \subset X$, we have $A_{d}^{++}=A_{\rho}^{++}$. Thus, if in addition, $d$ and $\rho$ determine the same bounded sets, then Theorem 3.6 guarantees that $\sigma_{d}=\sigma_{\rho}$. Since by Corollary 3.2, $\tau_{W_{\rho}} \subset \sigma_{\rho}$, we obtain $\tau_{\text {weak }} \subset$ $\sigma_{d}$.

For the other inclusion, we recall that for each open $V$, the set $V^{-}$belongs to each Wijsman topology [FLL, Proposition 2.1]. So, it remains to show that $\left(B^{c}\right)_{d}^{++} \in \tau_{\text {weak }}$, whenever $B$ is a closed and bounded subset of $X$. We dispose of some special cases. If $B=X$, then $\left(B^{c}\right)_{d}^{++}=\varnothing$, which is in each Wijsman topology. If $B=\{x\}$ for some $x$, then with respect to each $\rho,\left(B^{c}\right)_{\rho}^{++}=$ $\left(B^{c}\right)_{d}^{++}=\{F \in C L(X): \rho(x, F)>0\}$, which again is in $\tau_{W_{\rho}}$ for each $\rho$. It remains to consider the case that $B$ is not a singleton, and $B \neq X$.

Fix $A \in\left(B^{c}\right)_{d}^{++}$; we will produce $\rho \in \Delta, y_{0} \in X$, and $\delta>0$ such that

$$
A \in\left\{F \in C L(X): \rho\left(y_{0}, A\right)-\delta<\rho\left(y_{0}, F\right)\right\} \subset\left(B^{c}\right)_{d}^{++} .
$$

This would show that $\left(B^{c}\right)_{d}^{++}$contains a $\tau_{\text {weak }}$-neighborhood of each of its points.

Our metric $\rho: X \times X \rightarrow[0, \infty)$ will be of the form

$$
\rho(x, y)=\alpha d(x, y)+|d(x, B)-d(y, B)|,
$$


where $\alpha>0$. That $\rho$ and $d$ define the same uniformities follows from the $d$-uniform continuity of $x \rightarrow d(x, B)$. Since $\rho(x, y) \leq(\alpha+1) d(x, y), d$ bounded sets are $\rho$-bounded, and since $d(x, y) \leq \rho(x, y) / \alpha, \rho$-bounded sets are $d$-bounded. Thus, $\rho \in \Delta$. Recalling that $B$ consists of at least two points, the choice of $\alpha$ we make is $\alpha=D_{d}(A, B) / 4 \operatorname{diam} B$. Fix $y_{0} \in B$ and set $\delta=\alpha d\left(y_{0}, A\right)$. We intend to show that if $F \in C L(X)$ and $\rho\left(y_{0}, A\right)-\delta<$ $\rho\left(y_{0}, F\right)$, then $F \in\left(B^{c}\right)_{d}^{++}$.

First note that

$$
\rho\left(y_{0}, A\right)=\inf _{a \in A} \alpha d\left(y_{0}, a\right)+d(a, B) \geq \alpha d\left(y_{0}, A\right)+D_{d}(A, B)=D_{d}(A, B)+\delta .
$$

Thus, $\rho\left(y_{0}, F\right)>D_{d}(A, B)$. This means that for each $x \in F$, we have

$$
\alpha d\left(x, y_{0}\right)+d(x, B)>D_{d}(A, B) \text {. }
$$

We consider two cases for $x \in F$ : (i) $d\left(x, y_{0}\right) \leq 2 \operatorname{diam} B$; (ii) $d\left(x, y_{0}\right)>$ 2 diam $B$. In the first case, by the choice of $\alpha$, we get $\alpha d\left(x, y_{0}\right) \leq \frac{1}{2} D_{d}(A, B)$, so by $(*)$ we have $d(x, B)>\frac{1}{2} D_{d}(A, B)$. In the second case, since $y_{0} \in B$, we have $d(x, B) \geq \operatorname{diam} B$. Thus,

$$
D_{d}(F, B) \geq \min \left\{\frac{1}{2} D_{d}(A, B), \operatorname{diam} B\right\},
$$

and we have $F \in\left(B^{c}\right)_{d}^{++}$. This proves that $\left(B^{c}\right)_{d}^{++} \in \tau_{\text {weak }}$ for each closed and bounded subset $B$ of $X$, and we conclude that $\sigma_{d} \subset \tau_{\text {weak }}$.

One might guess from the previous results that there is a complete analogy between the $d$-proximal topology and the bounded $d$-proximal topology $\sigma_{d}$, upon replacing closed sets by closed and bounded sets in any theorem valid for $\tau_{\delta_{d}}$. Surprisingly, this is not the case, as we now show.

Theorem 3.8. Let $\langle X, d\rangle$ be a metric space. Then the $d$-proximal topology is the weak topology on $C L(X)$ determined by the family of excess functionals $\left\{e_{d}(\cdot, F): F \in C L(X)\right\}$.

Proof. Let $\tau_{\text {weak }}$ be the weak topology determined by $\left\{e_{d}(\cdot, F): F \in C L(X)\right\}$. Fix $A_{0}$ and $F$ in $C L(X)$. Upper semicontinuity of the excess functional $e_{d}(\cdot, F)$ at $A_{0}$ occurs if $e_{d}\left(A_{0}, F\right)=\infty$. Otherwise, $\left(S_{\varepsilon}\left[A_{0}\right]\right)^{++}$is a $\tau_{\delta_{d}}{ }^{-}$ neighborhood of $A_{0}$, and for each closed subset $A$ in this neighborhood, we have $e_{d}(A, F) \leq e_{d}\left(A_{0}, F\right)+\varepsilon$. Lower semicontinuity occurs if $e_{d}\left(A_{0}, F\right)=$ 0 . Otherwise, noting that $e_{d}\left(A_{0}, F\right)=\infty$ is possible, let $\alpha<e_{d}\left(A_{0}, F\right)$ be arbitrary, and choose $\varepsilon>0$ with $\alpha+\varepsilon<e_{d}\left(A_{0}, F\right)$. Choose $a_{0} \in A_{0}$ with $d\left(a_{0}, F\right)>\alpha+\varepsilon$. Then $S_{\varepsilon}\left[a_{0}\right]^{-}$is a $\tau_{\delta_{d}}$-neighborhood of $A_{0}$, and for each $A \in S_{\varepsilon}\left[a_{0}\right]^{-}$, we have $e_{d}(A, F)>\alpha$. This proves $\tau_{\delta_{d}}$-continuity of such an excess functional, so that $\tau_{\delta_{d}} \supset \tau_{\text {weak }}$.

For the other inclusion, suppose $A_{0} \in V^{-}$with $V$ open in $X$. Then there exists $a_{0} \in A_{0}$ and $\varepsilon>0$ such that $S_{\varepsilon}\left[a_{0}\right] \subset V$. With $F=\left\{x \in X: d\left(x, a_{0}\right) \geq\right.$ $\varepsilon\}$ we have

$$
A_{0} \in\left\{A \in C L(X): e_{d}(A, F)>0\right\} \subset V^{-} .
$$

If $A_{0} \in V^{++}$with $V$ open, there exists $\varepsilon>0$ with $S_{\varepsilon}\left[A_{0}\right] \subset V$. We then have

$$
A_{0} \in\left\{A \in C L(X): e_{d}\left(A, A_{0}\right)<\varepsilon\right\} \subset V^{++} .
$$

Together, these yield $\tau_{\delta_{d}} \subset \tau_{\text {weak }}$. 
Example. For closed and bounded sets $B$, the functional $A \rightarrow e_{d}(A, B)$ need not be $\sigma_{d}$-continuous or even $\tau_{\mathrm{aw}_{d}}$-continuous on $C L(X)$. For example, on the line with the usual metric, let $B=\{0\}$. Then $\{0\}=\tau_{\mathrm{aw}_{d}}-\lim \{0, n\}$, $\lim _{n \rightarrow \infty} e_{d}(\{0, n\}, B)=\infty$, whereas $e_{d}(\{0\}, B)=0$.

We do not intend to study the weak topology determined by $\left\{e_{d}(\cdot, B): B \in\right.$ $C L B(X)\}$, for such excess functionals fail to separate unbounded sets, and a non-Hausdorff topology results. It may be useful to study this weak topology on $C L B(X)$, but we do not consider subspaces of $C L(X)$ with induced topologies here.

\section{The Attouch-Wets and Hausdorff metric topologies AS WEAK TOPOLOGIES}

Of the three local presentations of the topology $\sigma_{d}$ we now concentrate on the one involving sets of the form $\Lambda_{A}\left[B ; \varepsilon ; x_{1}, x_{2}, \ldots, x_{k}\right]$, for it corresponds naturally to a combination of upper and lower halves of hyperspace topologies in the style of [FLL] or [AAB], as we now explain.

Recall that the Wijsman topology associated with a fixed metric $d$ is the weakest topology on $C L(X)$ such that for each $x \in X, d(x, \cdot): C L(X) \rightarrow$ $[0, \infty)$ is continuous. We may split this into its lower and upper halves [FLL, LL]:

$\tau_{W_{d}}^{-}=$the weakest topology on $C L(X)$ such that $\forall x, d(x, \cdot)$ is upper semicontinuous; $\tau_{W_{d}}^{+}=$the weakest topology on $C L(X)$ such that $\forall x, d(x, \cdot)$ is lower semicontinuous.

Note that the lower (resp. upper) half corresponds to upper (resp. lower) semicontinuity of distance functionals! A local base for $\tau_{W_{d}}^{-}$(resp. $\tau_{W_{d}}^{+}$) at $A \in$ $C L(X)$ consists of all sets of the form $\left\{F \in C L(X): \forall i \leq k, d\left(x_{i}, F\right)<\right.$ $\left.d\left(x_{i}, A\right)+\varepsilon\right\}$ (resp. $\left.\left\{F \in C L(X): \forall i \leq k, d\left(x_{i}, F\right)>d\left(x_{i}, A\right)-\varepsilon\right\}\right)$, where $\left\{x_{1}, x_{2}, \ldots, x_{k}\right\}$ is a finite subset of $X$ and $\varepsilon>0$. On the other hand, the Attouch-Wets topology splits into $\tau_{\mathrm{aw}_{d}}^{-}$and $\tau_{\mathrm{aw}_{d}}^{+}$, where a local base for $\tau_{\mathrm{aw}_{d}}^{-}$(resp. $\tau_{\mathrm{aw}_{d}}^{+}$) at $A$ consists of all sets of the form $\{F \in C L(X): \forall x \in B$, $d(x, F)<d(x, A)+\varepsilon\}$ (resp. $\{F \in C L(X): \forall x \in B, d(x, A)-\varepsilon<d(x, F)\}$, where $B$ is an arbitrary bounded subset of $X$, and $\varepsilon>0$. By Lemma 3.1, $\sigma_{d}=\tau_{W_{d}}^{-} \vee \tau_{\mathrm{aw}_{d}}^{+}$, where the supremum is taken in the lattice of hyperspace topologies. A natural dual for $\sigma_{d}$ is the dual bounded $d$-proximal topology $\sigma_{d}^{*}$ given by $\sigma_{d}^{*}=\tau_{W_{d}}^{+} \vee \tau_{\mathrm{aw}_{d}}^{-}$. In view of the above remarks, a local base for $\sigma_{d}^{*}$ at $A \in C L(X)$ consists of all sets of the form

$$
\begin{array}{r}
\Lambda_{A}^{*}\left[B ; \varepsilon ; x_{1}, x_{2}, \ldots, x_{k}\right]=\{F \in C L(X): \forall x \in B, d(x, F)<d(x, A)+\varepsilon, \\
\text { and } \left.\forall i \leq k, d\left(x_{i}, A\right)-\varepsilon<d\left(x_{i}, F\right)\right\},
\end{array}
$$

where $\varepsilon>0,\left\{x_{1}, x_{2}, \ldots, x_{k}\right\} \subset X$, and $B \subset X$ is bounded.

The proof of the following fact is left to the reader (see the proof of Lemma $3.1)$. 
Lemma 4.1. Let $\langle X, d\rangle$ be a metric space. Then a local base for the topology $\sigma_{d}^{*}$ at $A \in C L(X)$ consists of all sets of the form

$$
\begin{array}{r}
\Psi_{A}^{*}\left[B ; \varepsilon ; x_{1}, x_{2}, \ldots, x_{k}\right]=\left\{F \in C L(X): B \cap A \subset S_{\varepsilon}[F],\right. \text { and } \\
\left.\forall i \leq k, d\left(x_{i}, A\right)-\varepsilon<d\left(x_{i}, F\right)\right\},
\end{array}
$$

where $B$ is a bounded subset of $X, \varepsilon>0$, and $\left\{x_{1}, x_{2}, \ldots, x_{k}\right\}$ is a finite subset of $X$.

Theorem 4.2. Let $\langle X, d\rangle$ be a metric space. Then the topology $\sigma_{d}^{*}$ is the weakest topology $\tau$ on $C L(X)$ such that for each $B \in C L B(X), A \rightarrow e_{d}(B, A)$ is $\tau$ continuous on $C L(X)$.

Proof. We first show that each excess functional is $\sigma_{d}^{*}$-continuous. Fix $B \in$ $C L B(X)$. We write $\alpha=e_{d}(B, A)=\sup _{b \in B} d(b, A)$. Note that $\alpha$ is finite since $B$ is bounded. Let $\varepsilon>0$ be arbitrary, and choose $b_{0} \in B$ such that $d\left(b_{0}, A\right)>\alpha-\varepsilon / 2$. We claim that if $F \in \Psi_{A}^{*}\left[S_{\alpha+\varepsilon}[B] ; \varepsilon / 2 ; b_{0}\right]$, then $\left|e_{d}(B, A)-e_{d}(B, F)\right| \leq \varepsilon$.

Let $b \in B$ be arbitrary. There exists $a \in A$ with $d(b, a)<d(b, A)+\varepsilon / 2$. This means that $a \in S_{\alpha+\varepsilon}[B]$, so there exists $x \in F$ with $d(x, a)<\varepsilon / 2$. Thus,

$$
d(b, F) \leq d(b, x)<d(b, a)+\varepsilon / 2<d(b, A)+\varepsilon,
$$

so that

$$
e_{d}(B, F)=\sup _{b \in B} d(b, F) \leq \sup _{b \in B} d(b, A)+\varepsilon=\alpha+\varepsilon .
$$

On the other hand, we have

$$
e_{d}(B, F)=\sup _{b \in B} d(b, F) \geq d\left(b_{0}, F\right)>d\left(b_{0}, A\right)-\varepsilon / 2>\alpha-\varepsilon .
$$

This establishes $\sigma_{d}^{*}$-continuity of the excess.

Next let $\tau_{\text {weak }}$ be the weakest topology on $C L(X)$ such that for each closed and bounded set $B, A \rightarrow e_{d}(B, A)$ is continuous. First, notice that for fixed $x \in X, A \in C L(X)$, and $\varepsilon>0$,

$$
\begin{aligned}
& \{F \in C L(X): d(x, F)>d(x, A)-\varepsilon\} \\
& \quad=\left\{F \in C L(X): e_{d}(\{x\}, F)>e_{d}(\{x\}, A)-\varepsilon\right\} .
\end{aligned}
$$

This proves that $\tau_{W_{d}}^{+} \subset \tau_{\text {weak }}$. If $\tau_{\mathrm{aw}_{d}}^{-} \not \subset \tau_{\text {weak }}$, then there exists a net $\left\langle A_{\lambda}\right\rangle$ in $C L(X)$ convergent to $A \in C L(X)$ in $\tau_{\text {weak }}$ that fails to $\tau_{\text {aw }_{d}}^{-}$-converge to $A$. By Lemma 4.1, there exists a bounded set $B, \varepsilon>0$, and a cofinal set of indices $M$ in the underlying directed set such that for each $\lambda \in M$, there exists $a_{\lambda} \in A \cap B$ with $d\left(a_{\lambda}, A_{\lambda}\right) \geq \varepsilon$. Then $B_{0}=\operatorname{cl}\left\{a_{\lambda}: \lambda \in M\right\}$ is a bounded subset of $A$, $e_{d}\left(B_{0}, A\right)=0$, and $\lim \sup e_{d}\left(B_{0}, A_{\lambda}\right) \geq \varepsilon$. This contradicts the continuity of $F \rightarrow e_{d}\left(B_{0}, F\right)$ at $F=A$ with respect to $\tau_{\text {weak }}$. Thus, $\tau_{\text {aw }_{d}}^{-} \subset \tau_{\text {weak }}$, so that $\sigma_{d}^{*}=\tau_{W_{d}}^{+} \vee \tau_{\mathrm{aw}_{d}}^{-} \subset \tau_{\text {weak }}$.

The example following Theorem 3.4 shows equally well that $A \rightarrow e_{d}(F, A)$ need not be $\tau_{\mathrm{aw}_{d}}$-continuous for a fixed closed set argument $F$. Thus such an excess functional need not be $\sigma_{d}^{*}$-continuous. For gap functionals, the weak topology determined by $\left\{D_{d}(B, \cdot): B \in C L B(X)\right\}$ is unchanged if we replace $d$ by a uniformly equivalent metric $\rho$ with the same bounded sets. This is not the case for the weak topology determined by $\left\{e_{d}(B, \cdot): B \in C L B(X)\right\}$. 
Example. If $d$ is the zero-one metric on $Z^{+}$and $\rho$ is the metric defined by $\rho(1, i)=2$ for $i>1$ and $\rho(i, j)=1$ for $1<i<j$, then $\sigma_{d}^{*} \neq \sigma_{\rho}^{*}$. To see this, for $n \in Z^{+}$, let $A_{n}=\{1, n+1, n+2, \ldots\}$ and let $A=\{1\}$. It is easy to check that for each $i \in Z^{+}$, we have $\lim _{n \rightarrow \infty} d\left(i, A_{n}\right)=d(i, A)$ so that $A=\tau_{W_{d}}-\lim A_{n}$. Also, for each $\varepsilon>0$ and $n \in Z^{+}$we have $A \subset S_{\varepsilon}\left[A_{n}\right]$ so that $A=\tau_{\mathrm{aw}_{d}}^{-}-\lim A_{n}$. Together, these yield $A=\sigma_{d}^{*}-\lim A_{n}$. But $\rho(3, A) \neq$ $\lim _{n \rightarrow \infty} \rho\left(3, A_{n}\right)$, so that $\left\langle A_{n}\right\rangle$ fails to converge to $A$ in $\sigma_{\rho}^{*}$ or even in $\tau_{W_{\rho}}$.

Since $\tau_{W_{d}}^{+}$is weaker than $\tau_{\mathrm{aw}_{d}}^{+}$and $\tau_{W_{d}}^{-}$is weaker than $\tau_{\mathrm{aw}_{d}}^{-}$, we see that $\sigma_{d} \vee \sigma_{d}^{*}=\tau_{\mathrm{aw}_{d}}$. Combining Theorems 4.2 and 3.4, we get this characterization of the Attouch-Wets topology as a weak topology.

Theorem 4.3. Let $\langle X, d\rangle$ be a metric space. Then the Attouch-Wets topology on $C L(X)$ is the weakest topology $\tau$ on $C L(X)$ such that for each closed and bounded subset $B$ of $X$, both $A \rightarrow D_{d}(B, A)$ and $A \rightarrow e_{d}(B, A)$ are $\tau$ continuous.

Theorem 4.4. Let $\langle X, d\rangle$ be a metric space. Then the Attouch-Wets topology $\tau_{\mathrm{aw}_{d}}$ on $C L(X)$ is the weakest topology $\tau$ on $C L(X)$ such that for each metric $\rho$ uniformly equivalent to $d$ that determines the same bounded sets as $d$, and for each closed and bounded subset $B$ of $X, A \rightarrow e_{\rho}(B, A)$ is $\tau$-continuous.

Proof. Let $\Delta$ be the class of metrics described in the proof of Theorem 3.7. If $\rho \in \Delta$, then $\tau_{\mathrm{aw}_{d}}=\tau_{\mathrm{aw}_{\rho}}$ [BDC, Theorem 3.2], so that by Theorem 4.3, $A \rightarrow e_{\rho}(B, A)$ is $\tau_{\mathrm{aw}_{d}}$-continuous for each $\rho \in \Delta$ and each closed and bounded set $B$. On the other hand, the weak topology must contain the weak topology determined by $\left\{e_{\rho}(\{x\}, \cdot): x \in X, \rho \in \Delta\right\}=\{\rho(x, \cdot): x \in X, \rho \in \Delta\}$, which by Theorem 3.7, is $\sigma_{d}$. By Theorem 4.2, the weak topology also contains $\sigma_{d}^{*}$; so, it contains $\sigma_{d}^{*}$; so, it contains $\sigma_{d} \vee \sigma_{d}^{*}=\tau_{\mathrm{aw}_{d}}$.

Of course, we may also split the Hausdorff metric topology into its upper and lower parts (see, e.g., [KT, p. 39]). A local base for $\tau_{H_{d}}^{+}$(resp. $\tau_{H_{d}}^{-}$) at $A \in C L(X)$ consists of all sets of the form $\left\{F \in C L(X): F \subset S_{\varepsilon}[A]\right\}$ (resp. $\left.\left\{F \in C L(X): A \subset S_{\varepsilon}[F]\right\}\right)$, where $\varepsilon>0$. Dualizing the $d$-proximal topology $\tau_{\delta_{d}}=\tau_{W_{d}}^{-} \vee \tau_{H_{d}}^{+}$to get the dual d-proximal topology $\tau_{W_{d}}^{+} \vee \tau_{H_{d}}^{-}$, and keeping in mind Theorem 3.2 and 3.7 of [BLLN], we obtain results analogous to Theorems 4.2-4.4 with essentially the same proofs.

Theorem 4.5. Let $\langle X, d\rangle$ be a metric space. Then the topology $\tau_{W_{d}}^{+} \vee \tau_{H_{d}}^{-}$is the weakest topology $\tau$ on $C L(X)$ such that for each $F \in C L(X), A \rightarrow e_{d}(F, A)$ is $\tau$-continuous on $C L(X)$.

Note that the Hausdorff excess functional is, in this context, extended real valued.

Theorem 4.6. Let $\langle X, d\rangle$ be a metric space. Then the Hausdorff metric topology $\tau_{H_{d}}$ on $C L(X)$ is the weakest topology $\tau$ on $C L(X)$ such that for each $F \in$ $C L(X)$, both $A \rightarrow D_{d}(F, A)$ and $A \rightarrow e_{d}(F, A)$ are $\tau$-continuous.

In view of our characterization of the $d$-proximal topology in Theorem 3.8 in terms of excess functionals, we also have

Theorem 4.7. Let $\langle X, d\rangle$ be a metric space. Then the Hausdorff metric topology $\tau_{H_{d}}$ on $C L(X)$ is the weakest topology $\tau$ on $C L(X)$ such that for each $F \in$ $C L(X)$, both $A \rightarrow e_{d}(A, F)$ and $A \rightarrow e_{d}(F, A)$ are $\tau$-continuous. 
There is a transparent proof of Theorem 4.7 that we note. Evidently, such excess functionals are Lipschitz continuous with respect to Hausdorff distance, and $\tau$-continuity of the functionals with $F=A_{0}$ at $A=A_{0}$ gives $A_{0}=$ $H_{d}-\lim A_{\lambda}$ whenever $A_{0}=\tau$ - $\lim A_{\lambda}$. As noted earlier, $A \rightarrow e_{d}(A, B)$ for a fixed closed and bounded set $B$ need not be continuous with respect to the Attouch-Wets topology.

Reasoning as in the proof of Theorem 4.4, we obtain

Theorem 4.8. Let $\langle X, d\rangle$ be a metric space. Then the Hausdorff metric topology $\tau_{H_{d}}$ on $C L(X)$ is the weakest topology $\tau$ on $C L(X)$ such that for each metric $\rho$ uniformly equivalent to $d$ and for each closed subset $F$ of $X, A \rightarrow e_{\rho}(F, A)$ is $\tau$-continuous.

\section{MORE ON HIT-AND-MISS TOPOLOGIES AS WEAK TOPOLOGIES}

By Theorem 3.1 of [BLLN], the Vietoris topology on $C L(X)$ is generated by the family of distance functionals $\{\rho(x, \cdot): x \in X$ and $\rho$ is compatible with the topology on $X\}$. What happens if we restrict our metrics to those that determine the same class of bounded sets? First, a definition.

Definition. Let $\langle X, d\rangle$ be a metric space. Then the bounded Vietoris topology $\tau_{b V}$ on $C L(X)$ associated with the metric $d$ has as a subbase all sets of the form $V^{-}$where $V$ is open and all sets of the form $\left(B^{c}\right)^{+}$where $B$ is closed and bounded.

There is no loss in generality in requiring that the open sets in the above definition be bounded to achieve symmetry in the definition.

Theorem 5.1. Let $\langle X, d\rangle$ be a metric space, and let $\Sigma=\{\rho: \rho$ is a metric equivalent to $d$ that determines the same bounded sets as $d\}$. Then $\tau_{b V}$ is the weak topology on $C L(X)$ determined by $\{\rho(x, \cdot): x \in X, \rho \in \Sigma\}$.

Proof. Let $\tau_{\text {weak }}$ be the weak topology determined by the prescribed family of distance functionals. By Proposition 2.1 of [FLL], for each $\rho \in \Sigma, x \in X$, and $\alpha \geq 0$, the set $\{F \in C L(X): \rho(x, F)<\alpha\}$ is contained in $\tau_{b V}$. Now suppose $A \in\{F \in C L(X): \rho(x, F)>\alpha\}$. Choosing $\beta>\alpha$ with $\rho(x, A)>\beta$, we have

$$
A \in\{w \in X: \rho(x, w)>\beta\}^{+} \subset\{F \in C L(X): \rho(x, F)>\alpha\} .
$$

Together, these show $\tau_{\text {weak }} \subset \tau_{b V}$.

For the reverse inclusion, as in the proof of Theorem 3.7, it suffices to show that for each closed $d$-bounded set $B$ and each $A \in\left(B^{c}\right)^{+}$, there exists $\rho \in \Sigma$, $y_{0} \in X$, and $\delta>0$ with

$$
\left\{F \in C L(X): \rho\left(y_{0}, A\right)-\delta<\rho\left(y_{0}, F\right)\right\} \subset\left(B^{c}\right)^{+} .
$$

Again, this is trivial if $B$ is a singleton; so, we assume that $B$ has at least two points. First, separate $A$ and $B$ by a Urysohn function $\varphi$, and construct an equivalent metric $\rho_{1}$ given by $\rho_{1}(x, y)=d(x, y)+|\varphi(x)-\varphi(y)|$. Evidently, bounded sets are not changed, and $D_{\rho_{1}}(A, B) \geq 1$. With $\alpha=$ $D_{\rho_{1}}(A, B) / 4 \operatorname{diam} B$, our desired metric $\rho$ is given by

$$
\rho(x, y)=\alpha \rho_{1}(x, y)+\left|\rho_{1}(x, B)-\rho_{1}(y, B)\right| .
$$


Then $\rho \in \Sigma$. With $y_{0} \in B$ arbitrary and $\delta=\alpha \rho_{1}\left(y_{0}, A\right)$, the proof of Theorem 3.7 yields

$$
\left\{F \in C L(X): \rho\left(y_{0}, A\right)-\delta<\rho\left(y_{0}, F\right)\right\} \subset\left(B^{c}\right)_{\rho_{1}}^{++} \subset\left(B^{c}\right)^{+} .
$$

In view of Theorem 3.6, we have

Corollary 5.2. Let $\langle X, d\rangle$ be a metric space, and let $\Sigma=\{\rho: \rho$ is a metric equivalent to $d$ that determines the same bounded sets as $d\}$. Then the bounded Vietoris topology determined by the metric $d$ is the weak topology determined by the family of gap functionals $\left\{D_{\rho}(B, \cdot): B \in C L B(X), \rho \in \Sigma\right\}$.

By Theorem 4.8, the supremum of all Hausdorff metric topologies corresponding to compatible metrics for a metrizable space $X$ is the weak topology determined by all functionals of the form $A \rightarrow e_{d}(F, A)$ where $F$ ranges over the closed subsets of $X$ and $d$ ranges over the compatible metrics for the topology of $X$. This supremum topology, called the locally finite topology, admits an interesting hit-and-miss presentation, as described in [BHPV] (see also [NS]). For $\Omega$ a family of subsets of $X$, write $\Omega^{-}=\{F \in C L(X): \forall E \in \Omega$, $E \cap F \neq \varnothing\}$.

Definition. Let $X$ be a metrizable space. Then the locally finite topology $\tau_{\mathrm{lf}}$ on $C L(X)$ has as a subbase all sets of the form $\Omega^{-}$where $\Omega$ is a locally finite family of open subsets of $X$, and all sets of the form $V^{+}$where $V$ is an open subset of $X$.

Theorem 5.3. Let $X$ be a metrizable space. Then the locally finite topology on $C L(X)$ is the weak topology determined by the family $\left\{e_{d}(F, \cdot): F \in C L(X)\right.$ and $d$ is a compatible metric for the topology of $X\}$.

Proof. Apply Theorem 4.8 above and Theorem 2.1 of [BHPV].

We now develop a bounded analog for the last result, using very different arguments from those presented in [BHPV]. The reader is invited to construct a proof of Theorem 2.1 of [BHPV] along these lines. First, a definition.

Definition. Let $\langle X, d\rangle$ be a metric space. The bounded locally finite topology $\tau_{\text {blf }}$ on $C L(X)$ determined by $d$ has as a subbase all sets of the form $\Omega^{-}$ where $\Omega$ is a uniformly bounded locally finite family of open subsets of $X$, and all sets of the form $\left(B^{c}\right)^{+}$where $B \in C L B(X)$.

Theorem 5.4. Let $\langle X, d\rangle$ be a metric space. Then the bounded locally finite topology $\tau_{\text {blf }}$ determined by $d$ is the weakest topology $\tau$ on $C L(X)$ such that $A \rightarrow e_{\rho}(B, A)$ is $\tau$-continuous for each $B \in C L B(X)$ and for each compatible metric $\rho$ determining the same bounded sets as the initial metric $d$. Thus, $\tau_{\mathrm{blf}}$ is the supremum of the Attouch-Wets topologies corresponding to metrics that determine the same bounded subsets as $d$.

Proof. The last assertion is immediate from Theorem 4.4, upon proving the first. As usual, denote the weak topology described above by $\tau_{\text {weak }}$. We first show that $\tau_{\text {weak }} \subset \tau_{\text {blf }}$. To this end fix a closed and bounded set $B$ and a metric $\rho$ determining the same bounded sets as $d$. We will show that $A \rightarrow e_{\rho}(B, A)$

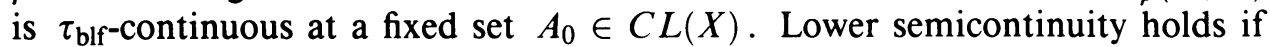
$e_{\rho}\left(B, A_{0}\right)=0$. Otherwise, fix $\varepsilon$ between 0 and $e_{\rho}\left(B, A_{0}\right)$, and choose $b \in B$ 
with $\rho\left(b, A_{0}\right)>e_{\rho}\left(B, A_{0}\right)-\varepsilon / 2$. Then $B_{0}=\left\{x: \rho(x, b) \leq \rho\left(b, A_{0}\right)-\varepsilon / 2\right\}$ is a closed bounded set disjoint from $A_{0}$, and if $A \in\left(B_{0}^{c}\right)^{+}$, then $e_{\rho}(B, A) \geq$ $\rho(b, A)>e_{\rho}\left(B, A_{0}\right)-\varepsilon$. Upper semicontinuity is harder. For each $b \in B$, choose $a_{b} \in A_{0}$ with $\rho\left(a_{b}, b\right)<\rho\left(b, A_{0}\right)+\varepsilon / 3$. Now let $E=\left\{a_{b}: b \in B\right\}$. Evidently, $E$ is bounded. By Zorn's lemma, there exists a maximal subset $E^{\prime}$ of $E$ such that for each $x$ and $y$ in $E^{\prime}$ we have $\rho(x, y) \geq \varepsilon / 3$. Clearly, the family of open balls $\Omega=\left\{S_{\varepsilon / 10}[x]: x \in E^{\prime}\right\}$ is uniformly bounded and locally finite; in fact, the family is discrete, i.e., each point in $X$ has a neighborhood meeting at most one element of the family. We claim that if $A \in \Omega^{-}$, then $e_{\rho}(B, A)<e_{\rho}\left(B, A_{0}\right)+\varepsilon$. Fix $b \in B$. By the maximality of $E^{\prime}$, there exists $x \in E^{\prime}$ such that $\rho\left(x, a_{b}\right)<\varepsilon / 3$. Since $A$ meets $S_{\varepsilon / 10}[x]$, we have $\rho(b, A)<$ $\rho\left(b, A_{0}\right)+\varepsilon / 3+\varepsilon / 3+\varepsilon / 10$. Thus,

$$
e_{\rho}(B, A)=\sup _{b \in B} \rho(b, A)<\sup _{b \in B} \rho\left(b, A_{0}\right)+\varepsilon=e_{\rho}\left(B, A_{0}\right)+\varepsilon .
$$

It remains to show that $\tau_{\text {weak }} \supset \tau_{\text {blf }}$. By Theorem 5.1, each set of the form $\left(B^{c}\right)^{+}$with $B$ closed and bounded is in $\tau_{\text {weak }}$, since distance functionals are excess functionals. Now let $\left\{V_{i}: i \in I\right\}$ be a bounded locally finite family of open sets, and suppose $A_{0} \in\left\{V_{i}: i \in I\right\}^{-}$. For each $i \in I$, choose $x_{i} \in A_{0} \cap V_{i}$. Although $i \rightarrow x_{i}$ need not be one-to-one, it is finite-to-one, by local finiteness. Now let $B=\left\{x_{i}: i \in I\right\}$. By local finiteness, we can choose for each $x \in B$ a number $\varepsilon_{x}>0$ such that the family $\left\{S_{2 \varepsilon_{x}}[x]: x \in B\right\}$ is discrete, and moreover, such that whenever $x \in V_{i}$ for some index $i$, then $S_{2 \varepsilon_{x}}[x] \subset V_{i}$. Let $V=\{w \in$ $\left.X: \forall x \in B, d(w, x)>\varepsilon_{x}\right\}$. Then $V$ is open so that $\{V\} \cup\left\{S_{2 \varepsilon_{x}}[x]: x \in B\right\}$ is a locally finite open cover of $X$. Let $\left\{g_{x}: x \in B\right\} \cup\{g\}$ be a partition of unity subordinated to this cover [En, p. 374], where of course $g_{x}^{-1}((0,1]) \subset S_{2 \varepsilon_{x}}[x]$ for $x \in B$ and $g^{-1}((0,1]) \subset V$. Then for each $w \in S_{\varepsilon_{x}}[x]$ we must have $g_{x}(w)=1$ whereas if $w \notin S_{2 \varepsilon_{x}}[x]$, we have $g_{x}(w)=0$. Evidently, the metric $\rho$ on $X$ defined by

$$
\rho(w, y)=d(w, y)+\sum_{x \in B}\left|g_{x}(w)-g_{x}(y)\right|
$$

is equivalent to $d$ and determines the same bounded sets, since the indexed sum on the right can be at most two. Clearly $A_{0} \in\left\{A \in C L(X): e_{\rho}(B, A)<1\right\}$ because $e_{\rho}\left(B, A_{0}\right)=0$. Also, if $e_{\rho}(B, A)<1$ holds for a closed set $A$, then for each $x \in B$ there exists $a \in A$ with $\rho(x, a)<1$. This means that

$$
1-g_{x}(a)=\left|g_{x}(x)-g_{x}(a)\right| \leq \rho(x, a)<1 \text {. }
$$

We conclude that $g_{x}(a)>0$ so that $A$ meets $S_{2 \varepsilon_{x}}[x]$. Since $x \in B$ was arbitrary, we conclude that $A$ meets $V_{i}$ for each $i \in I$. Thus,

$$
A_{0} \in\left\{A \in C L(X): e_{\rho}(B, A)<1\right\} \subset\left\{V_{i}: i \in I\right\}^{-},
$$

completing the proof that $\tau_{\text {weak }} \supset \tau_{\text {blf }}$.

\section{SOME FINAL OBSERVATIONS}

The reader may wonder why we have not considered compact sets as potential fixed left arguments in excess and gap functionals. The answer is simple, as may be quickly verified: no finer topology results using compact sets rather than singletons. 
Although it may seem unnatural, we can mix families of generating functionals corresponding to different classes of metrics and/or different classes of sets. Here are some typical outcomes; the simple details are left to the reader.

Theorem 6.1. Let $\langle X, d\rangle$ be a metric space. Then the weak topology on $C L(X)$ determined by the family of functionals $\left\{e_{d}(F, \cdot): F \in C L(X)\right\} \cup\left\{D_{d}(B, \cdot): B \in\right.$ $C L B(X)\}$ is $\tau_{\mathrm{aw}_{d}}^{+} \vee \tau_{H_{d}}^{-}$.

Theorem 6.2. Let $\langle X, d\rangle$ be a metric space. Then the weak topology on $C L(X)$ determined by the family of functionals $\left\{D_{d}(F, \cdot): F \in C L(X)\right\} \cup\left\{e_{d}(B, \cdot): B \in\right.$ $C L B(X)\}$ is $\tau_{H_{d}}^{+} \vee \tau_{\mathrm{aw}_{d}}^{-}$.

Theorem 6.3. Let $\langle X, d\rangle$ be a metric space. Then the weak topology on $C L(X)$ determined by the family of functionals $\left\{e_{d}(F, \cdot): F \in C L(X)\right\} \cup\{\rho(x, \cdot): x \in$ $X$ and $\rho$ equivalent to $d\}$ is the supremum of the Hausdorff metric topology associated with $d$ and the Vietoris topology.

We conclude with a table showing all the weak topologies obtainable using distance functionals, gap functionals, and excess functionals, using a single metric, a uniform class of metrics, or all metrics. For bounded set arguments, it is understood that the metrics are expected to determine the same class of bounded sets.

Table 1a. Weak topologies on $C L(X)$ induced by families of distance functionals, gap functionals, and excess functionals.

$\rho \approx d$ : the metrics $\rho$ and $d$ determine the same uniformity.

$\rho \underset{b d}{\approx} d:$ the metrics $\rho$ and $d$ determine the same uniformity and the same bounded sets.

$\rho \underset{b d}{\sim} d$ : the metrics $\rho$ and $d$ are equivalent and determine the same bounded sets.

\begin{tabular}{c|c|c|c} 
& $\{\rho(x, \cdot): x \in X\}$ & $\left\{D_{\rho}(F, \cdot): F \in C L(X)\right\}$ & $\left\{e_{\rho}(F, \cdot): F \in C L(X)\right\}$ \\
\hline$\rho=d$ & Wijsman topology & $d$-proximal topology & $\begin{array}{c}\text { dual } d \text {-proximal } \\
\text { topology }\end{array}$ \\
\hline$\rho \approx d$ & $d$-proximal topology & $d$-proximal topology & $\begin{array}{c}\text { Hausdorff metric } \\
\text { topology }\end{array}$ \\
\hline all $\rho$ & Vietoris topology & Vietoris topology & $\begin{array}{c}\text { locally finite } \\
\text { topology }\end{array}$ \\
\hline
\end{tabular}

Table $1 b$

\begin{tabular}{c|c|c|c} 
& $\{\rho(x, \cdot): x \in X\}$ & $\left\{D_{\rho}(B, \cdot): B \in C L B(X)\right\}$ & $\left\{e_{\rho}(B, \cdot): B \in C L B(X)\right\}$ \\
\hline$\rho=d$ & Wijsman topology & $\begin{array}{c}\text { bounded } d \text {-proximal } \\
\text { topology }\end{array}$ & $\begin{array}{c}\text { bounded dual } \\
d \text {-proximal topology }\end{array}$ \\
\hline$\rho \underset{b d}{\widetilde{\approx}} d$ & $\begin{array}{c}\text { bounded } d \text {-proximal } \\
\text { topology }\end{array}$ & $\begin{array}{c}\text { bounded } d \text {-proximal } \\
\text { topology }\end{array}$ & $\begin{array}{c}\text { Attouch-Wets } \\
\text { topology }\end{array}$ \\
\hline$\rho \widetilde{b d} d$ & $\begin{array}{c}\text { bounded Vietoris } \\
\text { topology }\end{array}$ & $\begin{array}{c}\text { bounded locally } \\
\text { finite topology }\end{array}$ & $\begin{array}{c}\text { bounded locally } \\
\text { finite topology }\end{array}$ \\
\hline
\end{tabular}




\section{REFERENCES}

[At] H. Attouch, Variational convergence for functions and operators, Pitman, New York, 1984.

[AW] H. Attouch and R. Wets, Quantitative stability of variational systems. I. The epigraphical distance, Trans. Amer. Math. Soc. 328 (1991), 695-729.

[AAB] H. Attouch, D. Azé, and G. Beer, On some inverse stability problems for the epigraphical sum, Nonlinear Anal. 16 (1991), 241-254.

[ALW] H. Attouch, R. Lucchetti, and R. Wets, The topology of the $\rho$-Hausdorff distance, Ann. Mat. Pura Appl. (to appear).

[AP] D. Azé and J.-P. Penot, Operations on convergent families of sets and functions, Optimization 21 (1990), 521-534.

[Be1] G. Beer, On Mosco convergence of convex sets, Bull. Austral. Math. Soc. 38 (1988), 239-253.

[Be2] _ Convergence of continuous linear functionals and their level sets, Arch. Math. 52 (1989), 482-491.

[Be3] _ Conjugate convex functions and the epi-distance topology, Proc. Amer. Math. Soc. 108 (1990), 117-126.

[Be4] _ _ An embedding theorem for the Fell topology, Michigan Math. J. 35 (1988), 239-253.

[Be5] _ Support and distance functionals for convex sets, Numer. Funct. Anal. Optim. 10 (1989), $15-36$.

[Be6] _ _ A Polish topology for the closed subsets of a Polish space, Proc. Amer. Math. Soc. 113 (1991), 1123-1133.

[Be7] _ Mosco convergence and weak topologies for convex sets and functions, Mathematika 38 (1991), 89-104.

[BB] G. Beer and J. Borwein, Mosco convergence and reflexivity, Proc. Amer. Math. Soc. 109 (1990), 427-436.

[BDC] G. Beer and A. Di Concilio, Uniform continuity on bounded sets and the Attouch-Wets topology, Proc. Amer. Math. Soc. 112 (1991), 235-243.

[BHPV] G. Beer, C. Himmelberg, K. Prikry, and F. Van Vleck, The locally finite topology on $2^{X}$, Proc. Amer. Math. Soc. 101 (1987), 168-172.

[BLLN] G. Beer, A. Lechicki, S. Levi, and S. Naimpally, Distance functionals and the suprema of hyperspace topologies, Ann. Mat. Pura Appl. (to appear).

[BL1] G. Beer and R. Lucchetti, Convex optimization and the epi-distance topology, Trans. Amer. Math. Soc. 327 (1991), 795-813.

[BL2] _- Well-posed optimization problems and a new topology for the closed subsets of a metrizable space, Rocky Mountain J. Math. (to appear).

[BF] J. Borwein and S. Fitzpatrick, Mosco convergence and the Kadec property, Proc. Amer. Math. Soc. 106 (1989), 843-849.

[CV] C. Castaing and M. Valadier, Convex analysis and measurable multifunctions, Lecture Notes in Math., vol. 580, Springer-Verlag, Berlin and New York, 1977.

[Co] B. Cornet, Topologies sur les fermés d'un espace métrique, Cahiers de mathématiques de la décision, vol. 7309, Université de Paris Dauphine, Paris, 1973.

[En] R. Engleking, General topology, Polish Scientific Publishers, Warsaw, 1977.

[Fe] J. Fell, A Hausdorff topology for the closed subsets of a locally compact non-Hausdorff space, Proc. Amer. Math. Soc. 13 (1962), 472-476.

[FLL] S. Francaviglia, A. Lechicki, and S. Levi, Quasi-uniformization of hyperspaces and convergence of nets of semicontinuous multifunctions, J. Math. Anal. Appl. 112 (1985), 347-370.

[KT] E. Klein and A. Thompson, Theory of correspondences, Wiley, New York, 1984.

[LL] A. Lechicki and S. Levi, Wijsman convergence in the hyperspace of a metric space, Bull. Un. Mat. Ital. 5-B (1987), 435-452.

[Mi] E. Michael, Topologies on spaces of subsets, Trans. Amer. Math. Soc. 71 (1951), 152-182.

[Mo] U. Mosco, Convergence of convex sets and of solutions of variational inequalities, Adv. in Math. 3 (1969), 510-585. 
[NS] S. Naimpally and P. Sharma, Fine uniformity and the locally finite hyperspace topology on $2^{X}$, Proc. Amer. Math. Soc. 103 (1988), 641-646.

[Pe] J.-P. Penot, The cosmic Hausdorff topology, the bounded Hausdorff topology, and continuity of polarity, Proc. Amer. Math. Soc. 113 (1991), 275-285.

[SP] P. Shunmugaraj and D. V. Pai, On stability of approximate solutions of minimization problems, Numer. Funct. Anal. Optim. (to appear).

[SZ1] Y. Sonntag and C. Zalinescu, Scalar convergence of convex sets, J. Math. Anal. Appl. 164 (1992), 219-241.

[SZ2] _- Set convergences. An attempt of classification, Proc. Internat. Conf. on Differential Equations and Control Theory (Iasi, Romania, August, 1990), Pitman Research Notes in Math. \#250, 1991, pp. 312-323.

[Wi] R. Wijsman, Convergence of sequences of convex sets, cones, and functions. II, Trans. Amer. Math. Soc. 123 (1966), 32-45.

Department of Mathematics, California State University, los Angeles, California 90032

E-mail address: gbeer@calstatela.edu

Department of Mathematics, University of California, Davis, California 95616

Current address: Dipartimento di Matematica, Via C. Saldini 50, 20133 Milano, Italy 